\title{
WILEY-VCH
}

1 DOI: 10.1002/ ((please add manuscript number))

2 Article type: Full Paper

\section{Associative Enhancement of Time Correlated Response to Heterogeneous Stimuli in a Neuromorphic Nanowire Device}

Curtis J. O'Kelly1,3, Jessamyn A. Fairfield1,3, David McCloskey2,3, John F. Donegan2,3 and John J. Boland1,3*

Dr. Curtis J. O'Kelly 1,3, Dr. Jessamyn A. Fairfield 1,3, Dr. David McCloskey 2,3, Prof. John F. Donegan 2,3 and Prof. John J. Boland 1,3

1 School of Chemistry, Trinity College Dublin, Dublin 2, Ireland

2 School of Physics, Trinity College Dublin, Dublin 2, Ireland

3 CRANN Institute, Trinity College Dublin, Dublin 2, Ireland

*E-mail: jboland@tcd.ie. Phone: +353 1-896-3140.

Keywords: Memory, Learning, Associative Memory, Neuromorphic hardware, Pulse Stimuli, Synapse

In spite of the strong interest in brain-like or neuromorphic computation, relatively few devices have emerged whose neuromorphic behavior is embedded in the hardware itself and not reliant on external programming of synaptic weights. We describe here a neuromorphic device based on $\mathrm{TiO}_{2}$ nanowire that exhibits an associative memory response to the time correlation between voltage and optical stimuli. Memristive characteristics are also observed with current-voltage sweeps showing hysteresis loops and continuum resistance levels. The nanowire device responds to heterogeneous voltage and optical pulse stimuli with spike-like neuromorphic outputs. Moreover, uncorrelated pulses produce a weak response, consistent with the interaction of coincident pulses with adsorbed and bulk oxygen in the surface depletion region, leading to a nonlinear enhancement in conductance. The strength of this learned enhancement depends on the both the time correlation and number of pulse stimuli, consistent with spike timing dependent plasticity. The nanowire devices presented have neural synapse-like properties that could serve as a building block for neuromorphic computation.

\section{Introduction}




\section{WILEY-VCH}

The human brain's ability to temporally associate multiple complex stimuli is a key requirement for neuromorphic learning. ${ }^{[1,2]}$ Although many biologically-inspired silicon-based technologies have reproduced basic sensory functions from sight to hearing, ${ }^{[3,4]}$ mimicking neural cognition and functionality in silicon has thus far relied on externally-set weighted outputs to demonstrate learning, an approach which suffers from large device footprints, memory-computation lag and high power consumption. ${ }^{[5-7]}$ In an ideal neuromorphic device, the synaptic weights between elements are modulated by electrical pulses from neighboring devices and sensitive to a range of heterogeneous stimuli. These synaptic weights are strengthened when pulses arrive at nearly the same time which produces a phenomenon known as spike timing dependent plasticity (STDP) and which leads to learning. ${ }^{[2]}$ Recent studies seeking to emulate this behavior have focused on an emerging class of resistance switching devices called memristors, whose non-volatile continuum conductance states closely imitate the analog response of individual biological synapses. ${ }^{[8-11]}$ Neuromorphic hardware based on ionic channels and resistive switching materials has also been shown to emulate synaptic weight augmentation in response to external stimuli. ${ }^{[10-14]}$ However, the devices reported to date respond to just a single type of stimulus, namely voltage pulses..$^{[15]}$

Here we show associative, time-dependent correlation between voltage and optical pulses in a nanoscale neuromorphic device comprised of an $\mathrm{Au}$-contacted $\mathrm{TiO}_{2}$ nanowire. The observed behavior is similar to synaptic weight augmentation, but for physically heterogeneous stimuli, demonstrating novel associative memory not previously shown for memristive devices. Uncorrelated stimuli produce a weak electrical response, while coincident stimuli create a temporary modification of the device properties that leads to an enhanced conductance. The enhancement level depends on the time correlation and number of pulse stimuli, emulating STDP and demonstrating neuromorphic learning in a solid state device. This device has the potential to broaden not only the memristor concept but also to form a novel neuromorphic 


\section{WILEY-VCH}

hardware platform sensitive to multiple physically dissimilar stimuli, and which may also have applications in optoelectronic computation. ${ }^{[16,17]}$

\section{Results and Discussion}

The response of single $\mathrm{TiO}_{2}$ nanowire devices to voltage and optical pulse stimuli was studied using the experimental setup in Figure 1a. As previously shown, the device is initially activated by applying a large voltage (typically $+10 \mathrm{~V}$ ) until the current attains a steady-state value to create a steady state population of charged dopants at the $\mathrm{Au}-\mathrm{TiO}_{2}$ interface that facilitates charge injection into the wire. ${ }^{[18]}$ The magnitude of charge injection is modulated by the population of oxygen vacancies at the metal interface lowering the tunneling barrier. The effect produces a memristive diode-like response linked directly to the history of voltage pulses applied to the device. The current response to a half-wave sinusoidal voltage input is shown in Figure $1 \mathrm{~b}$ and characterized by memristive hysteresis loops that evolve to higher conductance levels with each voltage sweep. The corresponding $I-V$ curve in Figure $1 \mathrm{c}$ is consistent with a neuromorphic resistive switch, displaying a continuum of conductance states. Similar devices have also displayed evolving conductance levels in response to a repeated voltage pulse stimulus, tunable memory levels, and a unique reset state. ${ }^{[18]}$ An important aspect of the device operation is the presence of a threshold for the onset of current rectification in either direction. This occurs below $\pm 3 \mathrm{~V}$ and is highlighted in the inset of Figure 1c. Now, building on the previously reported memory properties, optical pulse stimuli are added in addition toto the voltage stimuli previously reported.

The photocurrent $\left(I_{p c}=I_{\text {Measured }}-I_{\text {Dark }}\right)$ recorded during one $20 \mathrm{~s}$ laser pulse is shown in Figure 2a. The photocurrent response is initially linear but then saturates above $5 \mathrm{~V}$. The response of the device to uncorrelated $20 \mathrm{~s}$ voltage and optical pulses is shown in Figure 2b, in addition to a combined pulse where the voltage and optical stimuli are coincident. The hold 


\section{WILEY-VCH}

91 voltage is $+2 \mathrm{~V}$ and was maintained for $300 \mathrm{~s}$ prior to the voltage pulse $(\Delta V=0.75 \mathrm{~V})$ to establish the current baseline. Critically, both the hold voltage and peak pulse voltage are below the threshold for modifying device conductance. Whereas the responses to the uncorrelated pulses is modest, the coincident pulse elicits an exceptional response that is clearly larger than the sum of the responses to the individual pulses. The current enhancement can be computed from the additional charge $d Q=I d t$ generated during each voltage, optical and coincident pulse, which are $14 \mathrm{pC}, 6 \mathrm{pC}$ and $87 \mathrm{pC}$, respectively. Similar enhancement levels were observed in over 10 individual devices produced in separate fabrication runs, with representative data reported in the Supporting Information.

To quantify the enhanced conduction, we initially assume the properties of the material remain unchanged during the coincident pulse. The current density generated by a voltage pulse is determined by the density of electron (holes) $n(p)$, their respective mobilities $\mu_{n}$ and $\mu_{p}$, and the local electric field $E$, which scales as $V / L$, where $V$ is the applied voltage and $L$ the interelectrode separation. During the coincident pulse the increase in current may be expressed as a sum of the current generated during a voltage pulse $\Delta I_{e l}$ and an optical pulse $\Delta I_{p c}$ as:

$$
\Delta I_{c o}(V \rightarrow V+\Delta V, \text { optical })=\Delta I_{e l}(V \rightarrow V+\Delta V)+\Delta I_{p c}(V+\Delta V)^{\alpha}
$$

where alpha $\alpha$ is a scalar describing the degree of current nonlinearity during the coincident pulse. (See Supporting Information for a detailed derivation.) The non-linearity in the I-V response is expressed as $E \propto V^{\alpha}$. Assuming fixed geometry and no change in material properties the coincident pulse response should be the sum of the voltage and the optical pulse responses in Figure 2b, with the latter rescaled by the factor $I_{e l}(V+\Delta V) / I_{e l}(V)$, which is estimated to be a factor of $\sim 2$ on average for the devices measured based on the data in Figure $1 \mathrm{~b}$ (inset and caption). Applying Equation (1) to the data in Figure $2 \mathrm{~b}$, i.e., $16 \mathrm{pA} \neq(2.5+1 \mathrm{x}$ 


\section{WILEY-VCH}

116 2) $\mathrm{pA}$, demonstrates that there is a nonlinear increase in current and total charge $(87 \mathrm{pC} \neq(14$

$117+6 \times 2) \mathrm{pC}$ ) during coincident pulses. This enhancement is observed over many devices and is

118 likely due to a modification of the material properties, as discussed below.

119 We now show that the degree of modification depends on the number and time correlation of

120 the pulse stimuli. Figure 3a shows an alternating train of voltage and optical pulses with a time

121 delay $\Delta \mathrm{t}=20 \mathrm{~s}$. Although each pulse is identical to that employed in Figure 2c, there is a weak

122 interaction between the pulses that produces an enhancement in the current during a steady train

123 of voltage-only pulses with the same time spacing. The enhancement factor is $1.5-2$ when $\Delta \mathrm{t}=$

$12420 \mathrm{~s}$. When $\Delta \mathrm{t}=5 \mathrm{~s}$, this factor increases to $\sim 5$ and the enhancement is observed to gradually

125 increase as the current response reaches a steady state value of $5.5 \mathrm{pA}$ (Figure $3 \mathrm{~b}$ ). A train of

126 coincident pulses in Figure $3 \mathrm{c}$ produce the largest enhancements $(>20)$, which remains for a

127 certain time beyond the pulse duration directly analogous to synaptic weight change in

128 neuromorphic systems. Figure $3 \mathrm{~d}$ is a plot of the current enhancements as a function of pulse

129 time separation, in which the curve has been mirrored through $\Delta \mathrm{t}=0$ to reflect the fact that the

130 enhancement phenomenon is not dependent on the order in which the stimuli are delivered.

131 This sensitivity to time correlation, even for heterogeneous stimuli, is strikingly similar to the

132 STDP phenomenon found in biological systems, ${ }^{[19]}$ and that reported earlier for homogeneous

133 voltage impulses in memristors. ${ }^{[8,20]}$ In contrast to biological systems, however, we do not

134 observe inhibition when the order of the stimuli is reversed

135 We now explore the response of the device to probe pulses (optical and voltage) after the

136 device has experienced different numbers of coincident pulses. Figure 4 shows a pulse train

137 comprised of interleaved voltage (red) and optical (blue) pulses that allows for the direct overlap

138 of voltage and optical stimulus during every third pulse (orange). The initial individual voltage

139 and optical pulses produce a very modest response. The response to the first coincident pulse

140 is strong, but significantly the response to subsequent voltage and optical probe pulses (i.e. after

141 the coincident pulse) are also strengthened, similar to neural short term plasticity. ${ }^{[21]}$ Crucially, 


\section{WILEY-VCH}

142 each additional coincident pulse produces a growing response and is followed by an enhanced

143 response to the subsequent voltage and optical probe pulses. These data are consistent with a

144 learning behavior in which the device output grows following each coincident pulse. The

145 enhanced response to the subsequent voltage and optical probe pulses, the levels of which are

146 shown at the bottom of Figure 4a, demonstrates short-term associative memory - an effect

147 observed in all fabricated devices.

\section{3. Mechanism}

149 The time correlated enhancement in the device performance can be understood from the

150 known properties of $\mathrm{TiO}_{2}$ and its interaction with light. ${ }^{[22,23]}$ We begin by considering the

151 band alignment across the single nanowire device structure as shown in Figure 1a. The $\mathrm{Au}$

152 contact leads have a work function of $5.1 \mathrm{eV}$ and form Schottky barrier contacts with the wide

153 bandgap $\mathrm{TiO}_{2}$ nanowire, where the conduction band bends upwards due to the formation of a

154 depletion region as shown in Figure 4b. This Schottky barrier height can be further modified

155 by oxygen vacancy generation and adsorption of $\mathrm{O}_{2}$ onto the wire.

156 During device activation, oxygen vacancies readily form at the $\mathrm{TiO}_{2}$ interface with the $\mathrm{Au}$

157 metal contact under positive bias. ${ }^{[24-28]}$ The anodic conditions cause lattice oxygen $O_{O}^{\times}$to

158 react, forming positively charged oxygen vacancies $V_{O}^{\bullet \bullet}$ and releasing electrons into the

159 conduction band:[29]

$$
O_{O}^{\times} \rightleftarrows V_{O}^{\bullet}+2 e^{\prime}+\frac{1}{2} O_{2}(\mathrm{~g})
$$

Reaction (1)

163 The additional screening produced by these electrons reduces the width of the depletion

164 region and increases the transmission through the contact, resulting in activation of the

165 nanowire. However, the presence of $\mathrm{O}_{2}$ in the ambient and generated at the Au contact creates

166 an additional Schottky barrier due to reaction with electrons in the conduction band that results in the adsorption of $\mathrm{O}_{2}^{-}$species. ${ }^{[30-33]}$ 


\section{WILEY-VCH}

$$
e^{\prime}+O_{2}(\mathrm{~g}) \rightarrow \mathrm{O}_{2}^{-}(\mathrm{ads})
$$

Reaction (2)

170 This reaction occurs in wire regions with large populations of free electrons and hence

171 predominantly at the $\mathrm{Au}-\mathrm{TiO}_{2}$ interface. Charge depletion due to these adsorbed $\mathrm{O}_{2}{ }^{-}$species

172 increases the Schottky barrier at this interface. In the presence of light, however,

173 photogenerated holes are driven by the upward band bending towards the interface where they

174 are captured by the adsorbed $\mathrm{O}_{2}^{-}$species, resulting in the liberation of neutral $\mathrm{O}_{2}$. Based on

175 our device structure (see Figure 1) it is likely that most of the liberated $\mathrm{O}_{2}$ via reaction (1)

176 remains trapped at the $\mathrm{Au}-\mathrm{TiO}_{2}$ interface where it can in turn recombine with photogenerated

177 electrons via reaction (2). Thus the electrode-nanowire interface is an effective region for

178 recombination of photogenerated electron-hole pairs. This can be iunderstood by considering

179 the Schottky barrier hieight and width under diufferent bias conditions. For the forward-

180 biased Schottky barrier configuration considered here, the effective barrier height and the

181 depletion width decrease as the voltage is increased ${ }^{[34]}$ We therefore suggest that the large

182 band bending and broad depletion width present under low bias favours the recombination of

183 photogenerated carriers by sweeping holes towards the surface to react with O2- produced by

184 the adsorption of $\mathrm{O} 2$ with photogenerated electrons. This recombination process is suppressed

185 at higher applied biases and hence is responsible for the short term enhancement in

186 conductivity to coincident voltage and optical stimuli, which was observed in all devices.

187 Repeated coincident stimuli result in a steady build-up in the population of photogenerated

188 carriers resulting in the learning response described in Figure 4a.

189 The role of adsorbed oxygen at the metal/nanowire interface for the nonlinear conduction

190 enhancement mechanism and persistence with time can also be explored by the introduction

191 of a device capping layer. Repeating the same experiment as described in Figure $2 \mathrm{~b}$ on a

192 device capped with spincast SU-8 photoresist results in the data shown in Figure 5. Current

193 levels are increased in response to all pulse stimuli (voltage: $\Delta I_{e l}=2.75 \mathrm{pA}, \Delta Q_{e l}=16 \mathrm{pC}$; 
194 optical: $\Delta I_{p c}=5.32 \mathrm{pA}, \Delta Q_{p c}=25 \mathrm{pC}$; coincident: $\left.\Delta I_{C o}=65 \mathrm{pA}, \Delta Q_{C o}=330 \mathrm{pC}\right)$ but notably

195 the current response to the optical stimulus is considerably larger and longer lived when

196 compared to the actual pulse width. The pulse width is designated by the pale purple strip that

197 defines the actual time duration of the optical pulse. Isolating the device from atmospheric

198 oxygen improves the lifetime of the associative memory effect, enabling longer term

199 potentiation. The lifetime of the effect also depends on current magnitude and recombination

200 dynamics, as seen in Figures S2 and S3, and can be further extended via operation in vacuum

201 (Figure S4).

202 Mechanistically, the capping layer limits the supply of atmospheric oxygen from the ambient 203 and hence limits the recombination of the carriers generated during a coincident pulse.

204 Oxygen liberated from the material during the pulse remains trapped however and free to re-

205 adsorb on the surface. In contrast, devices without capping layers but measured under vacuum 206 conditions have little adsorbed oxygen and no capacity to replenish adsorbed oxygen from the 207 ambient, and in each case show a dramatically increased decay times for the photogenerated 208 current. The capping and vacuum experiments clearly show that the presence and availability 209 of oxygen dramatically affects the response of these devices. These oxygen mediated surface 210 reactions change the material functionality for a limit time period, enhancing the associative 211 memory response. These data strongly suggest that by controlling the surface environment 212 and/or functionalization it may be possible to improve neuromorphic performance.

\section{Conclusion}

We have introduced for the first time a single $\mathrm{TiO}_{2}$ nanowire device that is capable of 218 processing heterogeneous physical stimuli into an associative memory response. The device 219 also demonstrates time correlation that mimics the STDP learning processes found in biological systems. Although the memory is volatile in the present configuration, modifying surface 


\section{WILEY-VCH}

environment is shown to extend decay lifetimes. The enhancement effect due to the time correlation of the voltage and optical stimuli is robust and reproduced over several devices with the single-nanowire structure and is amenable to extending the number of inputs or feed forwarding the output of one device into another. The non-linear enhancement is postulated to arise from the increased concentration of photogenerated carriers during the voltage pulse and the mediating role adsorbed oxygen species have on the effective Schottky barrier height and carrier lifetime, These observations are supported by data taken on devices with a capping layer and those measured in vacuum. Given the simplicity of the device structure and the underlying nature of the Schottky barrier properties that control its operation, it will likely be possible to extend the range of physical stimuli (such as pressure, temperature, and magnetic field) that can be detected with temporal correlation. This is the first demonstration of a material which can process time-correlated heterogeneous inputs for neuromorphic applications, and the functionality developed here will likely prove useful in the development of neuromorphic sensing platforms.

\section{Experimental Section}

$\mathrm{TiO}_{2}$ nanowire devices were fabricated as reported previously using UV lithography, spray deposition and e-beam lithography. ${ }^{[18]}$ UV lithography is used to define large area contact pads before spray deposition of dilute solutions of $\mathrm{TiO}_{2}$ in deionized water. $\mathrm{TiO}_{2}$ wires (EMFUTUR) 50-100 $\mathrm{nm}$ in diameter and 5-20 $\mu \mathrm{m}$ in length were used to prepare devices. 80 $\mathrm{nm}$ of Au metal was deposited via electron beam evaporation to form contacts to the individual wires, with no adhesion layer used to ensure direct Au-wire contact. A Thor Labs SC10 shutter controller and SH1 beam shutter was used to modulate a $405 \mathrm{~nm}$ wavelength, $4.5 \mathrm{~mW}$ collimated laser diode that provides $0.5 \mathrm{~mW}$ of power at the wire. Voltage pulse and optical shutter sequences were controlled using a Keithley 4200-SCS parameter analyser, which also performs the current measurement. Vacuum measurements $\left(10^{-6}\right.$ torr $)$ were 


\section{WILEY-VCH}

248 performed in an ARS DE-204NI cryostat at room temperature. Wire devices are coated in Su-

2498 photoresist (Microchem) to form a UV transparent capping layer to isolate the device from

250 atmosphere. The SU-8 is dropped on the device, spun at low rpm (2000) and baked at $150^{\circ} \mathrm{C}$

251 for 5 minutes to cure the resist.

252

253

254

255

256

257

258

259

260

261

262

263

264

265

266

267

268

269

270

271

272

273

274

275

276

277

278

279

280

\section{Supporting Information}

Supporting Information is available from the Wiley Online Library or from the author.

\section{Acknowledgements}

C.J.O'K. designed and executed the experiments with the help of J.F. and J.B. C.J.O'K. and

J.F. analyzed the data and co-wrote the manuscript. D.McC. contributed to the development and optimization of the experimental setup as well as providing insight into data modeling and analysis. J.D. provided fruitful discussion. J.B. led the project, oversaw its development and contributed to the manuscript. The authors wish to acknowledge funding from the

European Research Council under Advanced Grant 321160. This publication has emanated from research supported in part by a research grant from Science Foundation Ireland (SFI)

AMBER Centre under Grant Number SFI/12/RC/2278.

Received: ((will be filled in by the editorial staff))

Revised: ((will be filled in by the editorial staff)) Published online: ((will be filled in by the editorial staff))

\section{References}

[1] W. Gerstner, R. Kempter, J. L. van Hemmen, H. Wagner, Nature 1996, 383, 76.

[2] G.-q. Bi, M.-m. Poo, Annual Review of Neuroscience 2001, 24, 139.

[3] J. Costas-Santos, T. Serrano-Gotarredona, R. Serrano-Gotarredona, B. LinaresBarranco, Circuits and Systems I: Regular Papers, IEEE Transactions on 2007, 54, 1444.

[4] W. Liu, A. G. Andreou, M. H. Goldstein, Jr., Neural Networks, IEEE Transactions on 1992, 3, 477.

[5] G. Indiveri, B. Linares-Barranco, T. J. Hamilton, A. van Schaik, R. Etienne-Cummings, T. Delbruck, S.-C. Liu, P. Dudek, P. Häfliger, S. Renaud, J. Schemmel, G. Cauwenberghs, J. Arthur, K. Hynna, F. Folowosele, S. SAÏGHI, T. Serrano-Gotarredona, J. Wijekoon, Y. Wang, K. Boahen, Frontiers in Neuroscience 2011, 5. 


\section{WILEY-VCH}

281 [6] B. V. Benjamin, G. Peiran, E. McQuinn, S. Choudhary, A. R. Chandrasekaran, J. M. 282 Bussat, R. Alvarez-Icaza, J. V. Arthur, P. A. Merolla, K. Boahen, Proceedings of the IEEE 2014, $283102,699$.

284 [7] P. A. Merolla, J. V. Arthur, R. Alvarez-Icaza, A. S. Cassidy, J. Sawada, F. Akopyan, B. 285 L. Jackson, N. Imam, C. Guo, Y. Nakamura, B. Brezzo, I. Vo, S. K. Esser, R. Appuswamy, B. 286 Taba, A. Amir, M. D. Flickner, W. P. Risk, R. Manohar, D. S. Modha, Science 2014, 345, 668. 287 [8] S. H. Jo, T. Chang, I. Ebong, B. B. Bhadviya, P. Mazumder, W. Lu, Nano Letters 2010, $28810,1297$.

289 [9] B. Linares-Barranco, T. Serrano-Gotarredona, L. A. Camuñas-Mesa, J. A. Perez290 Carrasco, C. Zamarreño-Ramos, T. Masquelier, Frontiers in Neuroscience 2011, 5.

291 [10] T. Hasegawa, T. Ohno, K. Terabe, T. Tsuruoka, T. Nakayama, J. K. Gimzewski, M. 292 Aono, Advanced Materials 2010, 22, 1831.

293 [11] S. Ambrogio, S. Balatti, F. Nardi, S. Facchinetti, D. Ielmini, Nanotechnology 2013, 24, 384012.

[12] S. La Barbera, D. Vuillaume, F. Alibart, ACS Nano 2015, 9, 941.

[13] S. Kyungah, K. Insung, J. Seungjae, J. Minseok, P. Sangsu, P. Jubong, S. Jungho, P. B. Kuyyadi, K. Jaemin, L. Kwanghee, L. Byounghun, H. Hyunsang, Nanotechnology 2011, 22, 254023.

[14] M. D. Pickett, G. Medeiros-Ribeiro, R. S. Williams, Nat Mater 2013, 12, 114.

[15] Y. V. Pershin, M. D. Ventra, Neural Netw. 2010, 23, 881.

[16] C. T. Phare, Y.-H. Daniel Lee, J. Cardenas, M. Lipson, Nat Photon 2015, 9, 511.

[17] G. T. Reed, G. Mashanovich, F. Y. Gardes, D. J. Thomson, Nat Photon 2010, 4, 518.

[18] C. O'Kelly, J. A. Fairfield, J. J. Boland, ACS Nano 2014, 8, 11724.

[19] D. O. Hebb, The Organization of Behavior: A Neuropsychological Theory, Taylor \& Francis Group, 2012.

[20] Y. V. Pershin, M. Di Ventra, Neural Networks 2010, 23, 881.

[21] T. Ohno, T. Hasegawa, T. Tsuruoka, K. Terabe, J. K. Gimzewski, M. Aono, Nat Mater 2011, 10, 591.

[22] M. A. Henderson, Surface Science Reports 2011, 66, 185.

[23] G. Liu, N. Hoivik, X. Wang, S. Lu, K. Wang, H. Jakobsen, Electrochimica Acta 2013, 93, 80 .

[24] D. S. Jeong, H. Schroeder, U. Breuer, R. Waser, Journal of Applied Physics 2008, 104, 123716.

[25] J. Joshua Yang, F. Miao, M. D. Pickett, D. A. Ohlberg, D. R. Stewart, C. N. Lau, R. S. Williams, Nanotechnology 2009, 20, 215201.

[26] D. H. Kwon, K. M. Kim, J. H. Jang, J. M. Jeon, M. H. Lee, G. H. Kim, X. S. Li, G. S. Park, B. Lee, S. Han, M. Kim, C. S. Hwang, Nature nanotechnology 2010, 5, 148.

[27] U. Diebold, Surf. Sci. Rep. 2003, 48, 53.

[28] N. Kazuki, Y. Takeshi, K. Masaki, O. Keisuke, K. Annop, R. Sakon, M. Gang, H. Mati, X. Bo, Z. Fuwei, H. Yong, K. Tomoji, Jpn. J. Appl. Phys. 2012, 51, 11 PE09.

[29] D. S. Jeong, H. Schroeder, R. Waser, Phys. Rev. B 2009, 79, 195317.

[30] M. S. T. Berger, O. Diwald, E. Knozinger, D. Panayotov, T. L. Thompson, J. T. Yates, Journal of Physical Chemistry B 2005, 6061.

[31] J. Zou, Q. Zhang, K. Huang, N. Marzari, The Journal of Physical Chemistry C 2010, $114,10725$.

[32] M. D. Rasmussen, L. M. Molina, B. Hammer, The Journal of Chemical Physics 2004, $120,988$.

[33] M. A. Henderson, W. S. Epling, C. L. Perkins, C. H. F. Peden, U. Diebold, The Journal of Physical Chemistry B 1999, 103, 5328. 
334
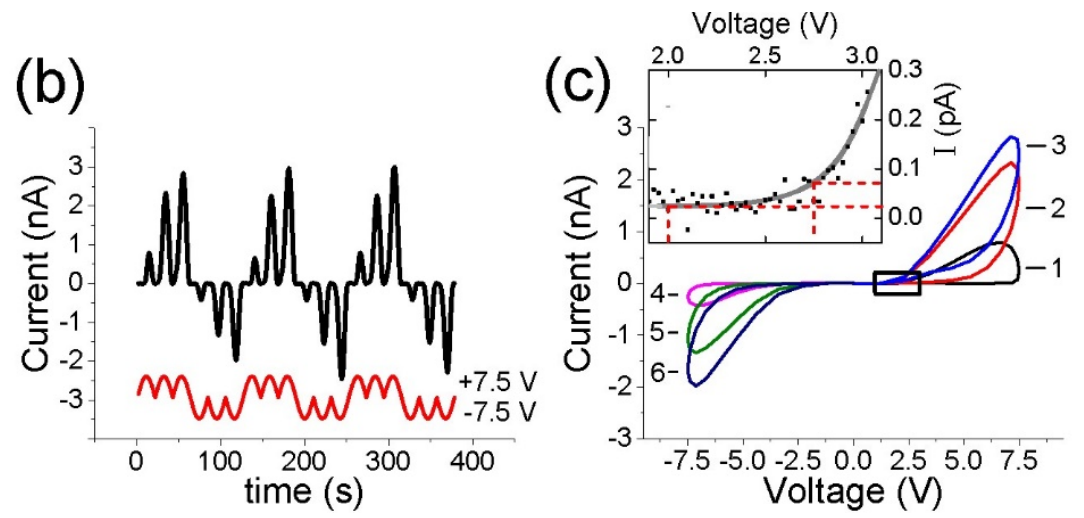

Figure 1. (a) An SEM image of a $\mathrm{TiO}_{2}$ nanowire (NW) with Au contacts (scale bar $1 \mu \mathrm{m}$ ), and a schematic of the experimental setup depicting a laser with a shutter linked to a controller and source measure unit. (b) Half wave sinusoidal voltage input produces an evolving current as a function of the number of input half waves. (c) The increase in conductance at one polarity may be selectively decreased or reset using opposite polarity inputs. The onset for current rectification occurs above $\pm 3 \mathrm{~V}$, as shown in inset (c), the dotted red lines are used to obtain the current at 2 and $2.75 \mathrm{~V}$ during sweep 1.

(a)

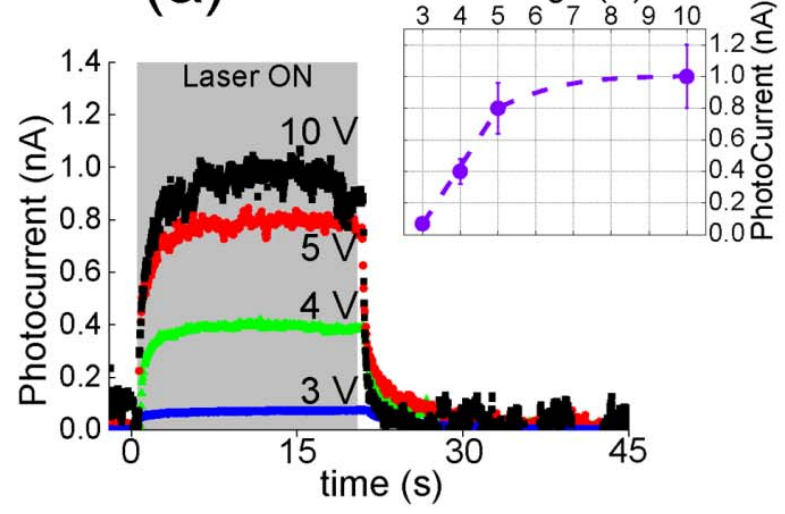

(b)

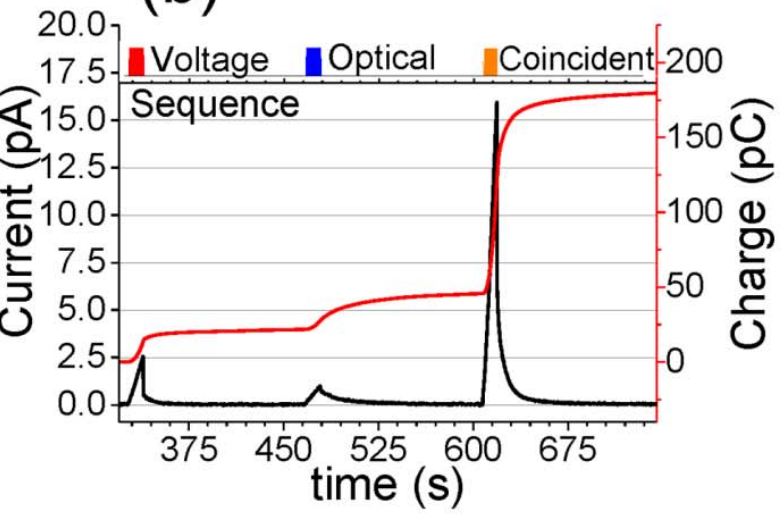

Figure 2. (a) Photocurrent produced in the nanowire device in response to a $20 \mathrm{~s}$ laser input at various holding voltage levels, inset shows the linear increase in photocurrent $( \pm 20 \%$ absolute value) up to $5 \mathrm{~V}$ with the photocurrent saturating at higher voltages. (b) Current response from separate heterogeneous voltage and optical pulses followed by a pulse consisting of both the optical and voltage pulse coincident on the nanowire simultaneously ( 2 $\mathrm{V}$ hold, pulse $\Delta 0.75)$. Each pulse is separated by a long dwell time at a hold voltage of $2 \mathrm{~V}$ to eliminate temporal coupling. The magnitude of current $\left(\Delta I_{c o}=16 \mathrm{pA}\right)$ and charge $\left(\Delta Q_{c o}=87\right.$ $\mathrm{pC})$ produced during the coincident pulse is not simply the linear addition of the current or charge produced by the individual voltage $\left(\Delta I_{e l}=2.5 \mathrm{pA}, \Delta Q_{e l}=14 \mathrm{pC}\right)$ and optical pulses $\left(\Delta I_{p c}=1 \mathrm{pA}, \Delta Q_{p c}=6 \mathrm{pC}\right)$. 
(a)
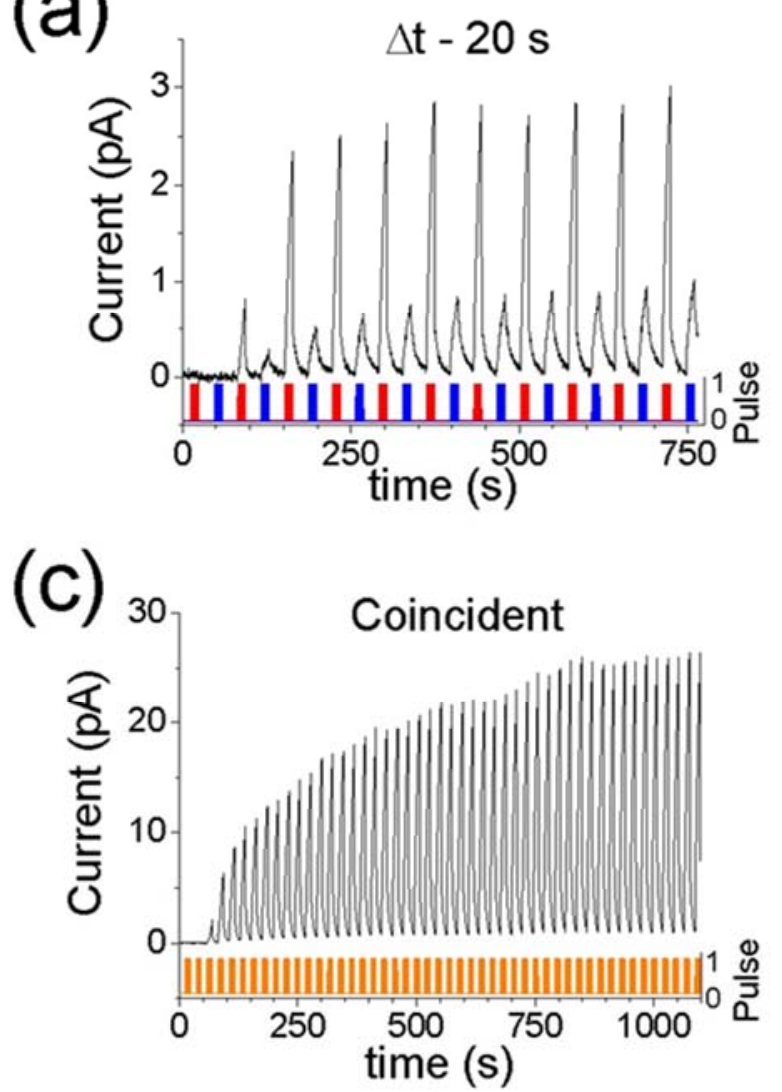
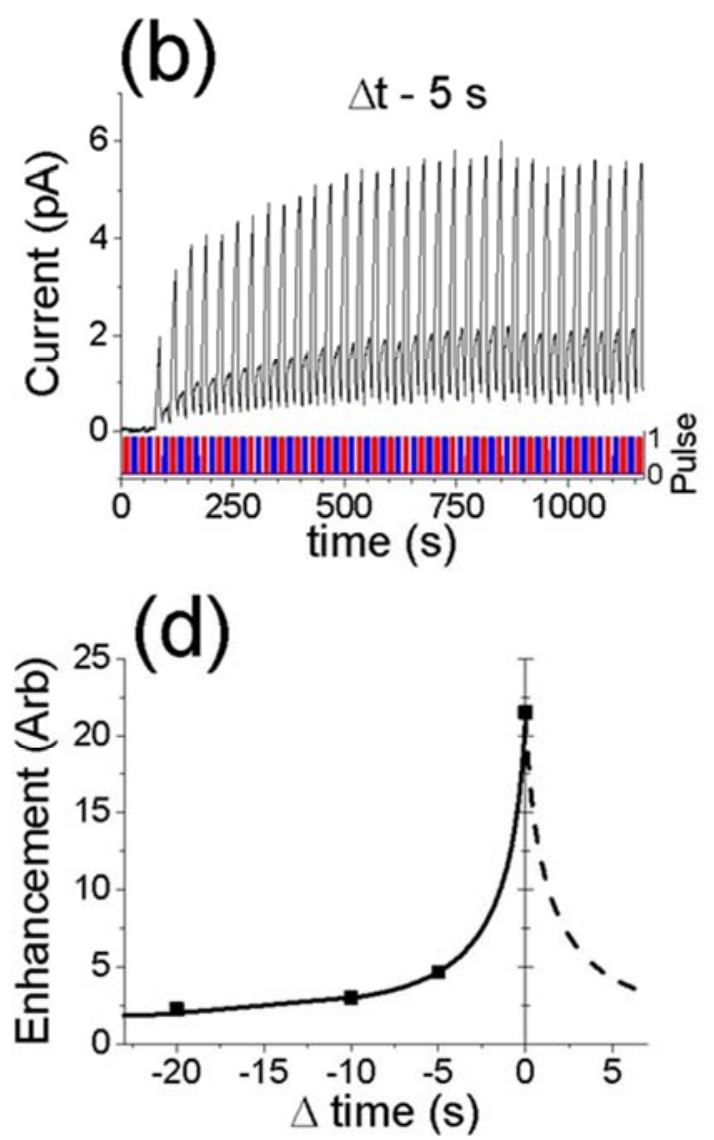

360

Figure 3. Pulse timing is critical to the interaction of separate pulse stimuli. For voltage and optical pulses separated by (a) 20 seconds, (b) 5 seconds, and (c) 0 seconds, the device conductance is augmented with a maximum augmentation for coincident pulses $(2 \mathrm{~V}$ holding bias, pulse $\Delta 0.75$ ). (d) A pseudo-plasticity strengthening graph analogous to STDP displaying the enhancement of device conductance as the time separation between pulses decreases.

(a)

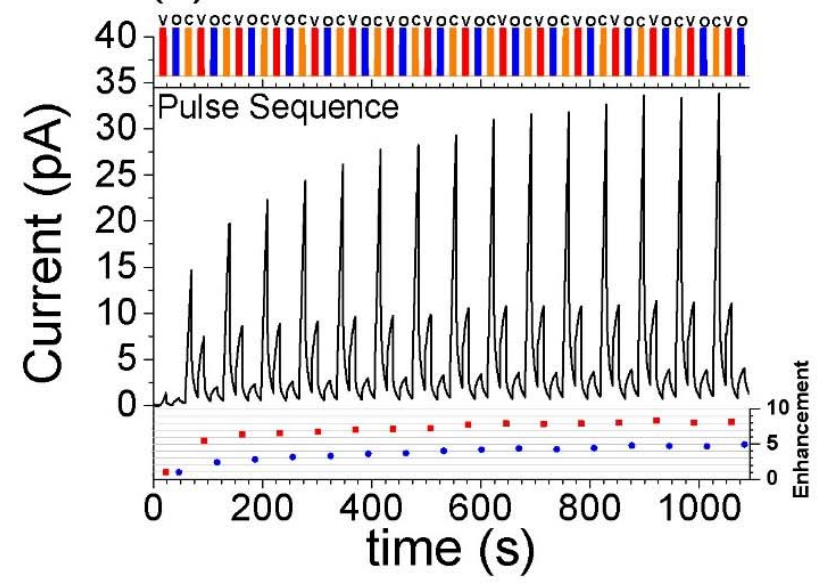

(b)

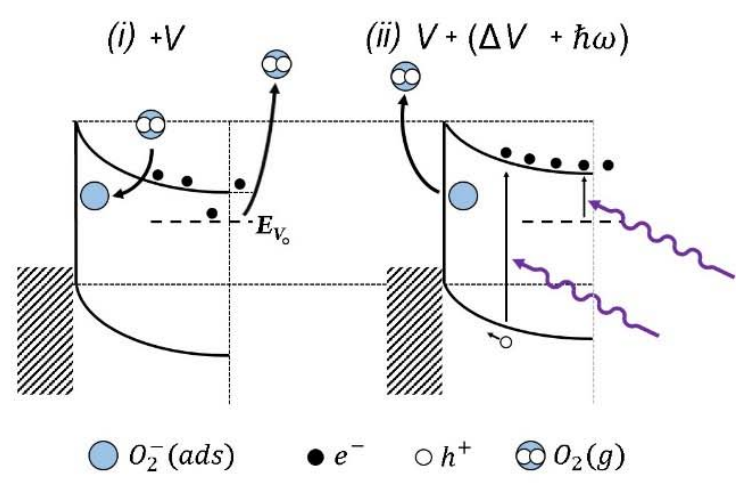

Figure 4. (a) A plot showing the device response to a series of coincident pulses that results in a growing and heightened current output, consistent with a learning phenomenon $(2 \mathrm{~V}$ holding bias, pulse $\Delta 0.75$ ). The lower panel shows the enhanced response to voltage (red) and optical (blue) probe pulses following each coincident pulse. The enhancement level 


\section{WILEY-VCH}

375 increases with the number of coincident pulses and demonstrates an associative memory

376 response, which is not observed following uncorrelated voltage and optical pulses. (b)

377 Schematic energy band diagram of the device interface at (i) $2 \mathrm{~V}$ hold condition and (ii)

378 during excitation with coincident stimuli comprised of a voltage $(\Delta V)$ and optical $(\hbar \omega)$ pulse.

379 Oxygen adsorbs at the $\mathrm{TiO}_{2} /$ metal interface, scavenging conduction band electrons and

380 augmenting the barrier height. Under the coincident pulse stimulus photogenerated holes combine with and release the adsorbed oxygen (see text) and when coupled with the increased voltage enhances charge injection into the electrode.

383

384

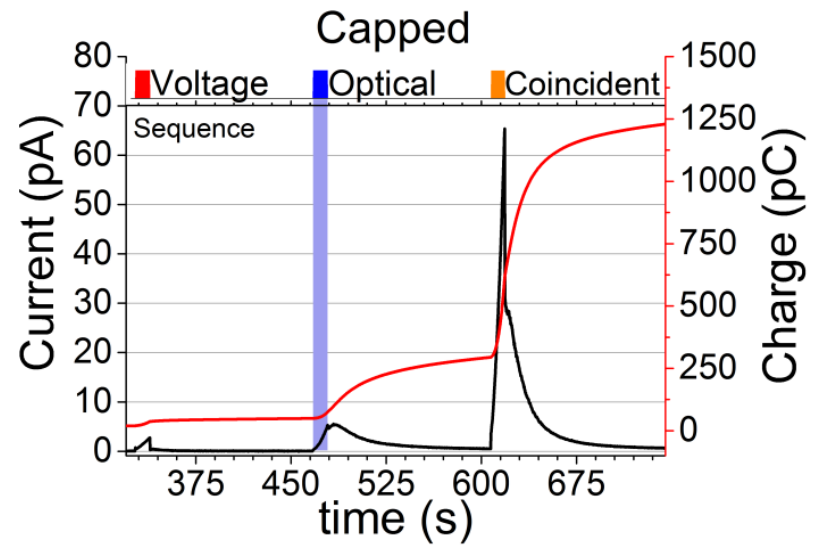

Figure 5. Isolating the device from atmospheric oxygen via a polymer capping layer has a profound impact on photogenerated current lifetime and the overall current levels produced by the device relative to the uncapped device, increasing the long term memory effect in response to pulse stimuli ( $2 \mathrm{~V}$ holding bias, pulse $\Delta 0.75$ ). 


\section{WILEY-VCH}

Table of Contents Entry:

A single $\mathrm{TiO} 2$ nanowire device demonstrates associative memory between two different pulse stimuli. Voltage pulses and optical UV laser light pulses both stimulate higher device current, but when the two pulses temporally coincide a nonlinear current enhancement occurs, similar to spike-timing dependent plasticity and caused by oxygen reactions within the depletion region of the device.

Keywords: Memory, Learning, Associative Memory, Neuromorphic hardware, Pulse Stimuli, Nanowire

Curtis J. O'Kelly1,3, Jessamyn A. Fairfield1,3, David McCloskey2,3, John F. Donegan2,3 and John J. Boland1,3*

Associative Enhancement of Time Correlated Response to Heterogeneous Stimuli in a Neuromorphic Nanowire Device

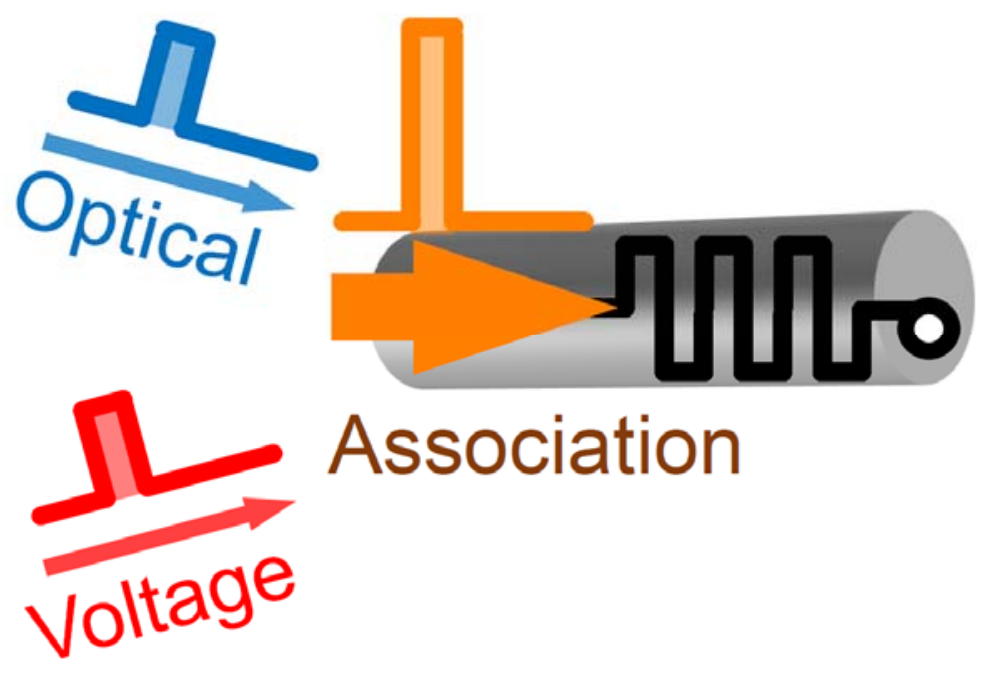




\section{WILEY-VCH}

Copyright WILEY-VCH Verlag GmbH \& Co. KGaA, 69469 Weinheim, Germany, 2013.

\section{Supporting Information}

Associative Enhancement of Time Correlated Response to Heterogeneous Stimuli in a Neuromorphic Nanowire Device

Curtis J. O'Kelly', , Jessamyn A. Fairfield ${ }^{1,3}$, David McCloskey ${ }^{2,3}$, John F. Donegan ${ }^{2,3}$ and John J. Boland ${ }^{1,3 *}$

\section{S1. Additional Voltage and Optical Enhancement Data}
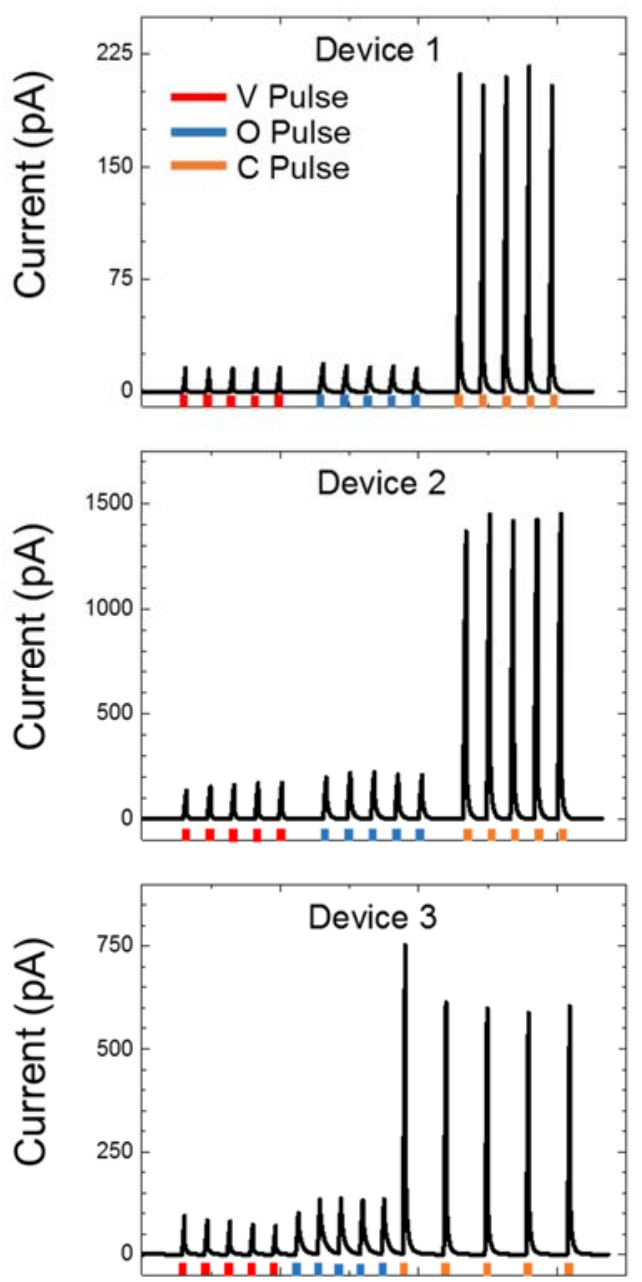

Time (s)

Figure S1. Coincident pulses increase current in all similar devices in a non linear addition of the separate voltage and optical pulses. Three separate devices are shown to display the same reproducible behavior in response to coincident pulses composed of voltage and optical pulses applied at the same time. 


\section{WILEY-VCH}

Table S1. The relative increase in current produced during separate voltage pulses and optical pulses are shown below.

\begin{tabular}{|l|l|l|}
\cline { 2 - 3 } \multicolumn{1}{l|}{} & Voltage Scaling & Optical Scaling \\
\hline Device 1 & 13.96 & 12.47 \\
\hline Device 2 & 9.21 & 6.74 \\
\hline Device 3 & 7.84 & 4.93 \\
\hline
\end{tabular}

\section{S2. Non-Linear Enhancement Derivation}

We initially assume the properties of the system remain unchanged during the coincident pulse, that current generated under the voltage pulse is due to the change in local electrical field and the photocurrent generated is from the separation of electrons and holes in the depletion region. The current density generated by a voltage pulse is determined by the density of electron (holes) $n(p)$, their respective mobilities $\mu_{n}$ and $\mu_{p}$, and the local electric field $E$, which scales as $V / L$, where $V$ is the applied voltage and $L$ the inter-electrode separation. For our fixed device geometry we can account for non-linearity in the current-voltage response by expressing $E \propto$ $V^{\alpha}$, where $\alpha$ is a scalar describing the degree of nonlinearity. The geometry-normalized current increase ( $\left.\Delta I_{e l}\right)$ (units: ampere per length) generated by a voltage pulse $\Delta V$ can be written as:

$$
\Delta I_{e l}(V \rightarrow V+\Delta V)=e\left[n \mu_{n}+p \mu_{p}\right] \cdot\left[(V+\Delta V)^{\alpha}-V^{\alpha}\right]
$$

The current response to an optical pulse $\left(\Delta I_{p c}\right)$ at a fixed voltage $V$ results in the generation of additional electrons $(\Delta n)$ and holes $(\Delta p)$ that increase the photocurrent by:

$$
\Delta I_{p c}(V)=e\left[\Delta n \mu_{n}+\Delta p \mu_{p}\right] \cdot V^{\alpha}
$$

During the coincident pulse, these photogenerated carriers experience an increased electric field due to the simultaneous voltage pulse. The increase in current can then be written as the sum of the equations $\mathrm{S} 1$ and $\mathrm{S} 2$ at the voltage pulse $\Delta V$ : 


\section{WILEY-VCH}

$$
\Delta I_{c o}(V \rightarrow V+\Delta V, o p t)=\Delta I_{e l}(V \rightarrow V+\Delta V)+\Delta I_{p c}(V+\Delta V)^{\alpha}
$$

449 Therefore, assuming no change in material properties, the coincident pulse response should be 450 the sum of the measured independent voltage and the optical pulse responses shown in Figure $4512 \mathrm{~b}$ of the main text. The factor $I_{e l}(V+\Delta V) / I_{e l}(V)$ is scaled to the voltage pulse $\Delta V$ as the 452 additional photocurrent conventionally increases at higher voltage. However even when the 453 photocurrent is scaled to the voltage pulse level as estimated to be a factor of $\sim 2$ based on the 454 data in Figure 1b(inset), there remains a large difference in the measured and calculated current. Equation (3) yields $2.5+1 \times 2=4.5 \Delta I_{c o}$ which is considerably less than the measured $\Delta I_{c o}=$ $16 \mathrm{pA}$. In the main text the difference is also presented as charge or the time integral of the current measured during each pulse to demonstrate the sharp current rise is not a short but large peak. This derivation and numerical calculation supports nonlinear current enhancement in the material under coincident pulse stimuli.

\section{S3. Persistence of Associative Memory}

The associative memory enhancement effect demonstrated in the present device is volatile. After multiple coincident pulses normal current level responses to voltage pulse stimuli are recovered after a period of 300-400 s. This is demonstrated in Figure S1, which shows a device with 5-20 coincident pulses applied to it prior to holding a bias of $2.75 \mathrm{~V}$ for an extended period. clearly over time. In all cases it takes approximately 300-400 s after the training coincident pulses are applied before the current levels reach normal levels similar to those without coincident pulses applied. The measured half-life (time taken for the enhanced current

471 magnitude to drop by half the its initial value following the coincident pulses) of the memory 472 association effect is indicated for each data and reveal that the memory persistence decreases 473 as the current magnitude increases. This volatility stems from the recombination of atmospheric 


\section{WILEY-VCH}

oxygen and charge carriers generated during the coincident pulse stimulus. At greater current magnitude and therefore higher charge concentration at the device interface the rate of recombination is accelerated. (a)

(b)

5 Prior Coincident Pulses 10 Prior Coincident Pulses
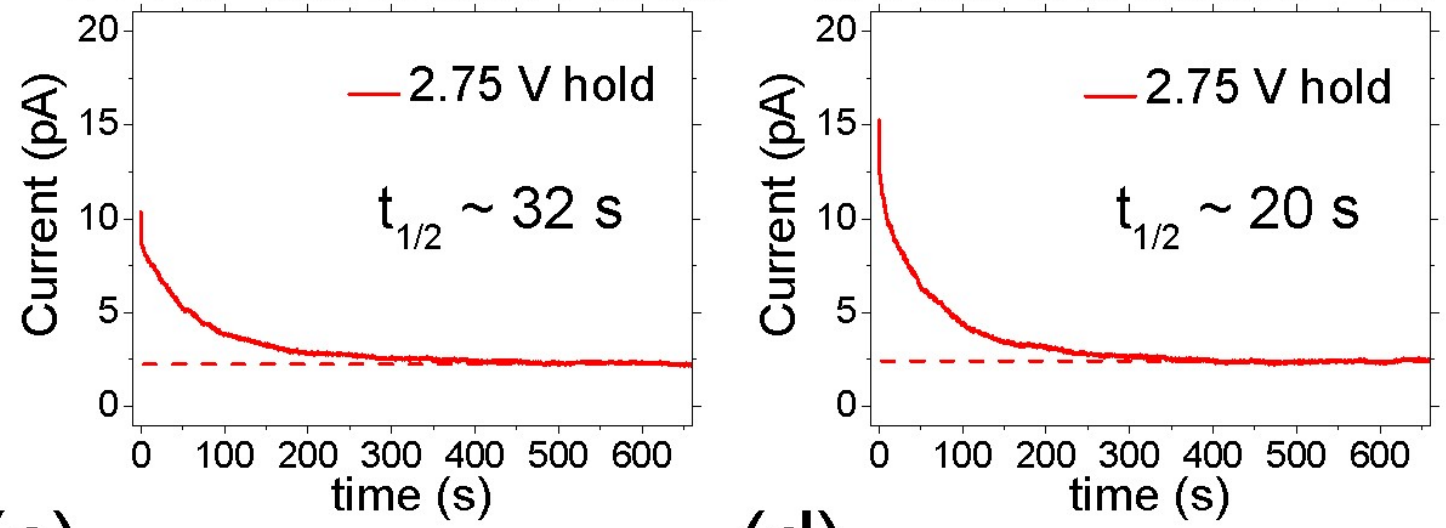

(d)
478

479

480

481

482

483

484
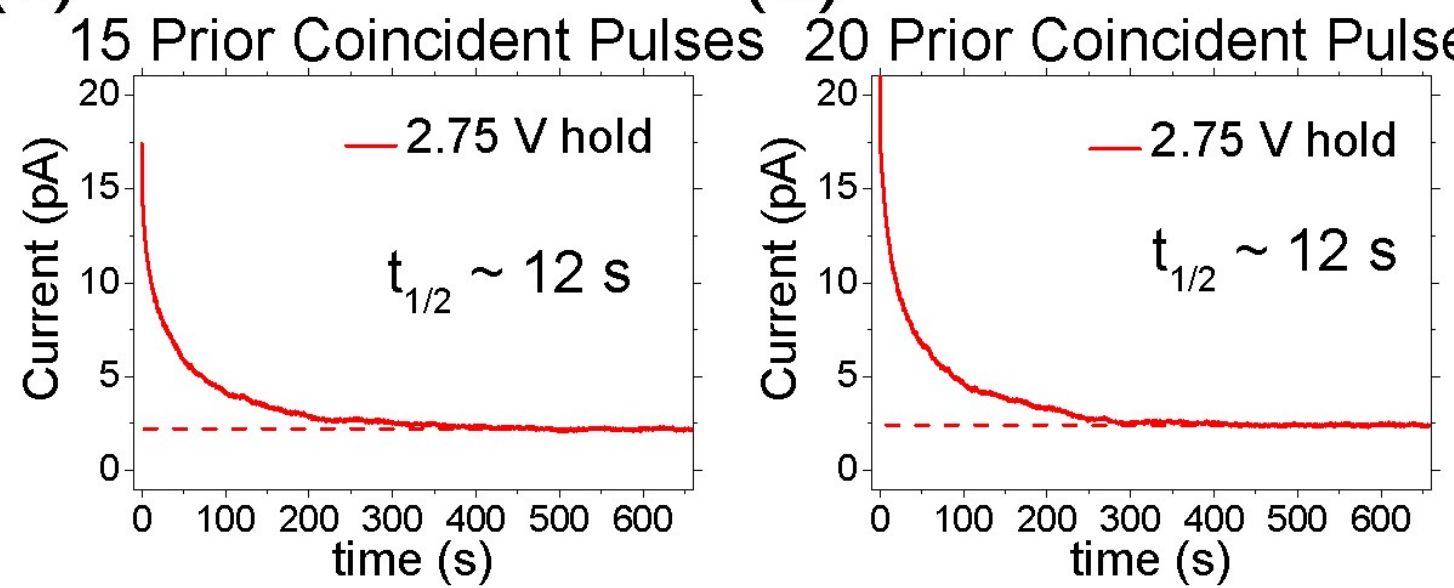

Figure S2. Memory association enhancement persistence as a function of the number of applied coincident pulses. Current enhancement persists longer with lower current magnitudes and decays faster as the current generated from greater number of coincident pulses increases as shown in the decrease in half life of the persistent current. THE NUMBER OF PULSES IS HARD TO NOTICE AT THE TOP OF THESE FIGS 
WILEY-VCH
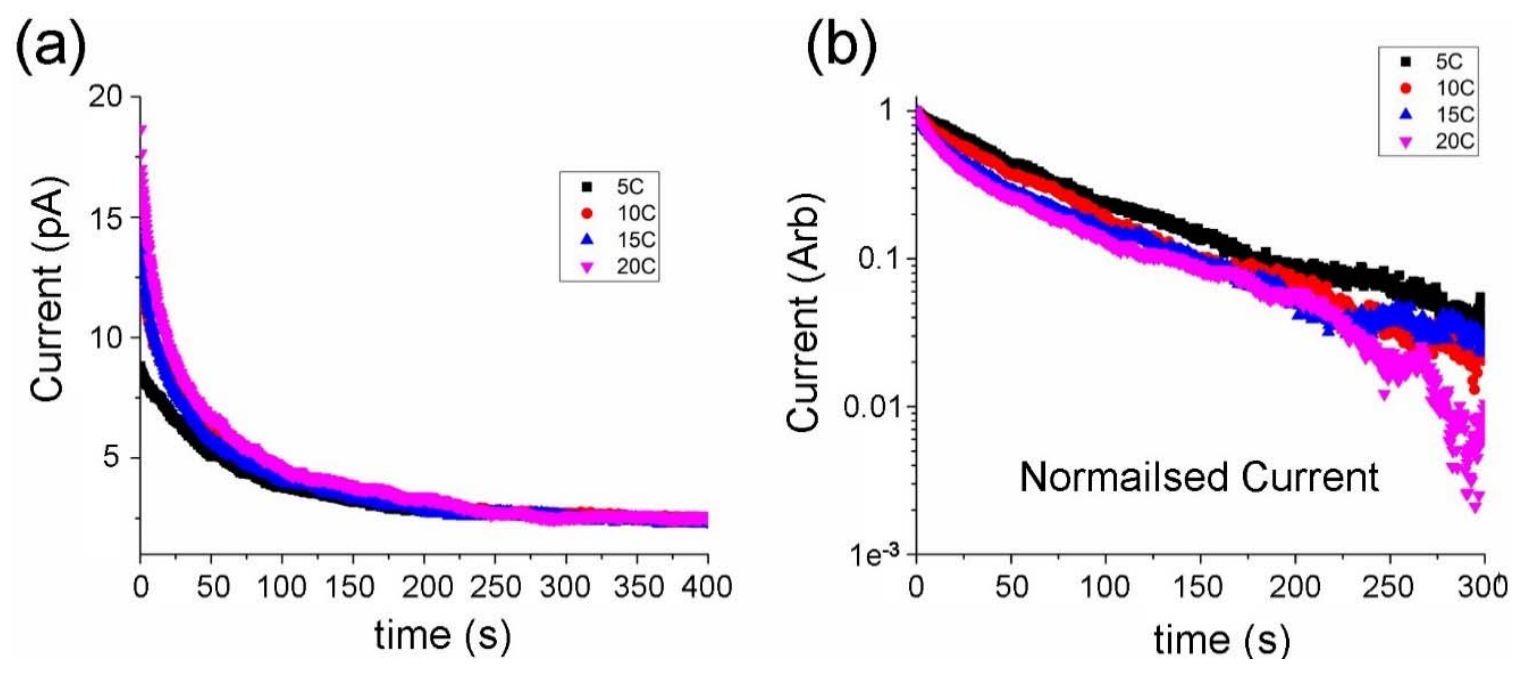

489 Figure S3. (a) A plot showing the current decay following 5 to 20 coincident pulses. (b) 490 Currents normalised and plotted on a log scale, the divergence from a straight line single 491 exponential decay becomes more apparent for larger numbers of applied coincident pulses.

493 The decay in persistent current levels does not fit a simple exponential decay for standard 494 persistent photocurrent recombination. In Figure S2 (a) the decay in the current after the 495 coincident pulses are applied are mapped onto a single plot. The rapid decrease in current at 496 higher current levels seems to support a more complicated bi-exponential decay with fast and 497 slow time constants. In Figure S2 (b) the current decay is normalized and plotted on a 498 logarithmic scale. If the decay was purely exponential these data would follow a straight line, 499 however we observe a clear shift in linearity as the number of coincident pulses applied to the 500 device increases. 


\section{WILEY-VCH}

501

502

503

504

505

506

507

508

509

510

511

512

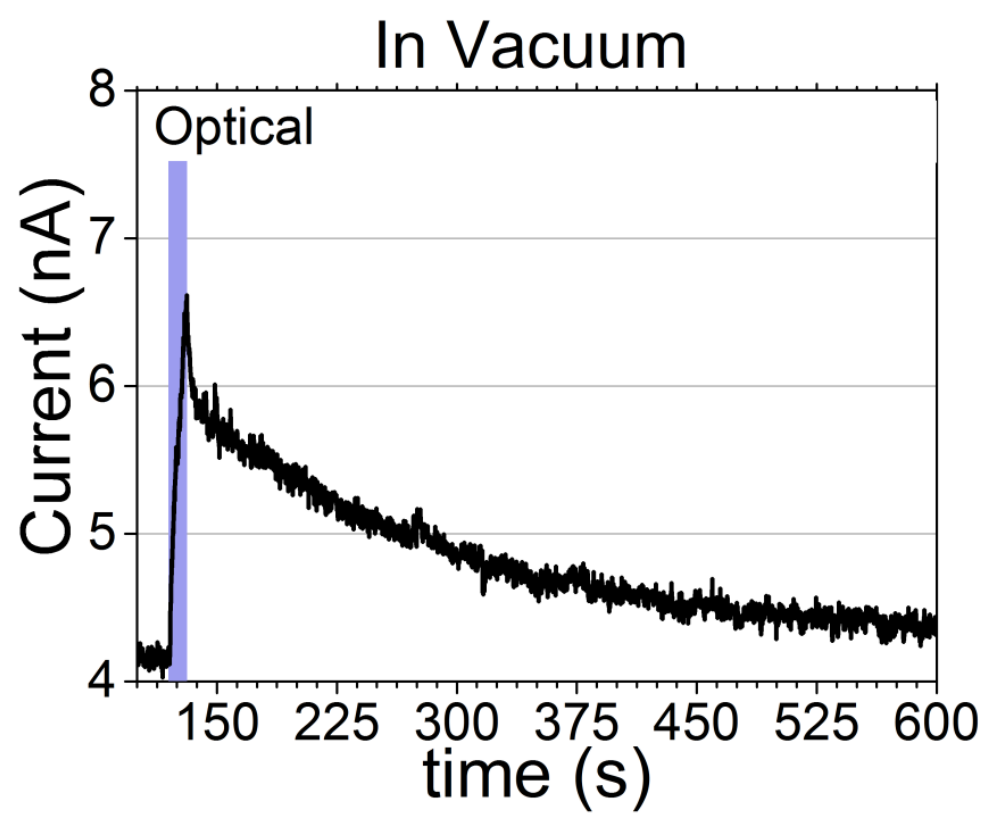

Figure S4. An optical pulse applied while the device is under vacuum $\left(10^{-6}\right.$ torr $)$ results in a much longer decay than the same measurement under atmosphere (see Figure S1), again implicating surface oxygen reactions. The holding bias voltage was $5 \mathrm{~V}$ which accounts for the larger photocurrent than was observed at a $2 \mathrm{~V}$ holding bias. NO data on simultaneous pulsing in vacuum??? 